\title{
Karnofsky Performance Status 60
}

National Cancer Institute

\section{Source}

National Cancer Institute. Karnofsky Performance Status 60. NCI Thesaurus. Code C105712.

Requires occasional assistance, but is able to care for most personal needs. 